\title{
Morphine and nicotine addiction and withdrawal influence baroreflex sensitivity and blood pressure in two-kidney one clip hypertensive $(2 \mathrm{~K} 1 \mathrm{C})$ rats
}

\author{
Zeinivand $\mathrm{M}^{1}$, Shamsizadeh $\mathrm{A}^{1}$, Pourshanazari AA ${ }^{1,2}$, Hassanshahi $\mathrm{G}^{3}$, Allatavakoli $\mathrm{M}^{1}$ \\ Physiology-Pharmacology Research Center, Rafsanjan University of Medical Sciences, Rafsanjan, Iran. \\ m_zeinivand2000@yahoo.com
}

\begin{abstract}
Objective: This study aimed to investigate the effects of addiction to morphine and nicotine as well as their withdrawal on both baroreflex sensitivity and blood pressure in hypertensive rats.

Methods: In this experimental study 40 male rats were divided into two main groups as follows: in group I, hypertensive rats received saline for 8 weeks; in group II, hypertensive rats were treated with morphine and nicotine for 8 weeks. At the end of 8 weeks group II rats were divided into four sub-groups including, 3 sub-groups of those were put on drug withdrawal protocol. At the end of experiment, blood pressure, heart rate, plasma renin activity (PRA), serum NO concentration and baroreflex sensitivity (BRS) were measured.

Results: Results demonstrated that BP and BRS were significantly lower in addicted to morphine and nicotine hypertensive rats compared to control $(p<0.05)$. Addiction withdrawal (in morphine and nicotine withdrawal rats) completely reversed BP and BRS to the pre-addiction levels $(p<0.05)$. Withdrawal in the only nicotine treated group lowered BP and BRS compared to group that had received morphine and nicotine together $(p<0.05)$. Conclusion: Results of current study may propose simultaneous morphine and nicotine withdrawal can prevent cardiovascular complications raised due to withdrawal (Fig. 5, Ref. 58). Text in PDF www.elis.sk.

Key words: hypertension, baroreflex sensitivity, morphine, nicotine.
\end{abstract}

The arterial baroreflex is defined as one of the most important physiological mechanisms involves in homeostasis of blood pressure (BP) (1). Baroreflex sensitivity (BRS) damage is a marker of autonomic dysfunction and plays a major role in the long-term development of arterial hypertension and related complications (2). It a pears that the function of arterial baroreflex is involved in the regulation of cardiovascular function $(3,4)$. Simultaneous elevated sympathetic nervous system and renin-angiotensin-aldosterone system modulates several biological changes including vascular changes, arterial stiffness, and increased BP eventually causing myocardial hypertrophy (5). Which further incorporate to a vicious cycle of hypertension and related complications. The baroreceptor reflex serves blood pressure buffer system for damping short-term variations of arterial pressure (1). Due to their complex rest in any new MAP baroreceptors have minor importance on chronic (6). On this light evidences indicated that long-term morphine ad-

'Physiology-Pharmacology Research Center, Rafsanjan University of Medical Sciences, Rafsanjan, Iran, ${ }^{2}$ Department of Physiology, Isfahan University of Medical Sciences, Isfahan, Iran, and ${ }^{3}$ Molecular Medicine Research Centre, Rafsanjan University of Medical Sciences, Rafsanjan, Iran

Address for correspondence: A. Asghar Pourshanazari, Department of Physiology, Hezar-Jerib Street. Isfahan University of Medical Sciences, 8174673461 Isfahan, Iran.

Phone: +98. 311. 7922435, Fax: +98. 311. 6688597

Acknowledgement: This study was financially supported by a grant from Rafsanjan University of Medical Sciences in collaboration with Isfahan University of Medical Sciences. diction may change the performance of these centers. Morphine in parallel with peripheral vasodilatation down-regulate systemic blood pressure (BP). This property of morphine is accompanied by inhibition of baroreceptor and depressed respiratory reflexes and sometimes causes cardiac arrest as well as respiratory problems (7). It assumed that the opioid actioncenter is located at the brain stem at primary baroreceptor synapses in the nucleus of the solitary tract. This region is concentrated with opioid containing fibers and opiate receptors $(8,9)$. The $\mathrm{BP}$, systemic vascular resistance and heart rate show acute and prolonged increases induced by cigarette smoking $(10,12)$. This happens following stimulation of the adrenaline and noradrenalin release in the sympathetic nerve system by nicotine $(10,12)$. Thus, a generalized peripheral adrenergic receptors stimulation is possibly in charge of the acute cardiovascular effects of smoking $(13,14)$. The impaired arterial baroreceptor function and other multiple factors, elevate associated cardiovascular risk factors of smoking (15). It is now well evidenced that the risk of cardiovascular diseases reduce immediately after leaving of cigarette smoking (16). Changes BP, heart rate (HR), and autonomic nervous function are thought to be at least in part responsible for the rapid reduction in the risk of cardiovascular diseases subsequent to quitting (17).

Due to the changes in BRS during hypertension (18), administration of morphine (19) and nicotine (20). May be useful Thus, the aim of this study was to evaluate the effect of chronic hypertension and concurrent administration of morphine and nicotine on $\mathrm{BRS}$ and BP and investigate whether if withdrawal of morphine 
and nicotine effect on improve of baroreflexe sensitivity and blood pressure. We asked whether if cardiovascular disorders are raised by simultaneous withdrawal of morphine and nicotine or these compounds act independently to run these disorders.

\section{Material and methods}

\section{Animals}

Forty male Wistar rats (aged 10-12 weeks old and weighting 200-250 g) were purchased from Pasteur Institute of Iran. Rats were kept in animal house with $12 \mathrm{~h}$ light/dark cycle and a temperature of $20-25{ }^{\circ} \mathrm{C}$. The animals had free access to tap water and commercial rat chow during experiment. Of period Ethical committee of Rafsanjan University of Medical Sciences approved the study protocol.

\section{Experimental protocol}

Following one week of getting familiar with animal house, male hypertensive rats were randomly divided in to two groups as follows: Group (1): received solvent of saline for 8 weeks via injection ( $\mathrm{n}=8$ ); Group (2): received $3 \mathrm{mg} / \mathrm{kg}$ of morphine and $0.01 \mathrm{mg} / \mathrm{kg}$ of nicotine 8 weeks via injection $(\mathrm{n}=32)$. At the end of 8 weeks groups (2) were divided into 4 sub-groups that 3 subgroups $(n=24)$ of those fit in drug withdrawals. The first sub-group was only starved from morphine $(n=8)$. Second sub-group was only starved from nicotine $(\mathrm{n}=8)$. The third sub-group was started from both morphine and nicotine $(n=8)$. Subsequent to 24 hours of withdrawal, naloxone $(2 \mathrm{mg} / \mathrm{kg}$ ) was injected. The withdrawal signs (body shakes, jumping, climbing, writhing, teeth chattering, irritability, ejaculation, ptosis and diarrhea) within $30 \mathrm{~min}$ after the injections of naloxone were evaluated.

\section{Induction of hypertension with 2K1C goldblatt method}

Initially rats were anesthetized using intraperitoneally ketamine hydrochloride $(60 \mathrm{mg} / \mathrm{kg})$ and xylazine $(7.5 \mathrm{mg} / \mathrm{kg})$. The left kidney was exposed via flank incision and a silver clip with an internal gap of $0.2 \mathrm{~mm}$ was located around the renal artery. To control the risk of infections, rats received a profilactic dose of penicillin $\mathrm{G}$ (25000 IU/rat) after surgery. Systolic and diastolic blood pressure was measured once a week by a tail-cuff plethysmography under light ether anesthesia. At the end of 8 weeks treatment, animals were anesthetized and direct blood pressure was measured by a catheter (PE50) which was inserted into femoral artery.

\section{Plasma renin activity}

The PRA was measured using Gamma coat PRA Radioimmunoassay kit (DiaSorinInc). PRA was measured after withdrawal in hypertensive rats.

\section{Serum NO measurement}

Serum NO concentrations were measured by Griess reagentkit (Promega, Madison, WI, Cat\#G2930) by spectrophotometer at a wavelength of $540 \mathrm{~nm}$ using standard curve as previously described (21).

\section{Baroreflex test}

The baroreflex was tested with a press or dose of phenylephrine (PE-bolus- $8 \mu \mathrm{g} / \mathrm{kg}$ IV; Sigma Chemical) (22). The baroreflex was calculated as the derivation of HR in function of the MAP variation $(\triangle \mathrm{HR} / \triangle \mathrm{MAP})(23)$. There was an interval of at least 15 minutes between the infusions to allow the recovery of basal values (18).

\section{Statistical analysis}

We applied one-way ANOVA for comparison of data between groups. Data are reported as mean \pm SE. $p$ value less than 0.05 was considered significant.

\section{Result}

\section{Evaluation of blood pressure and heart rat}

The systolic blood pressure (SBP), diastolic blood pressure (DBP) and mean arterial pressure (MAP) measurement in hypertensive rats showed that co-administration of morphine and nicotine in hypertensive rats significantly reduced BP in study groups when compared with control $(\mathrm{p}<0.05)$. In addicted rats withdrawal (specific in morphine + nicotine withdrawal) compared to nicotine withdrawal and co-administration of morphine and nicotine completely reversed BP to control group level ( $\mathrm{p}<0.05$ ) (Fig 1). HR was not significantly differed between groups (Fig 2).

\section{Measurement of PRA}

The PRA levels were decrees in nicotine withdrawal animals compared to control $(\mathrm{p}<0.05)$ (Fig. 3).

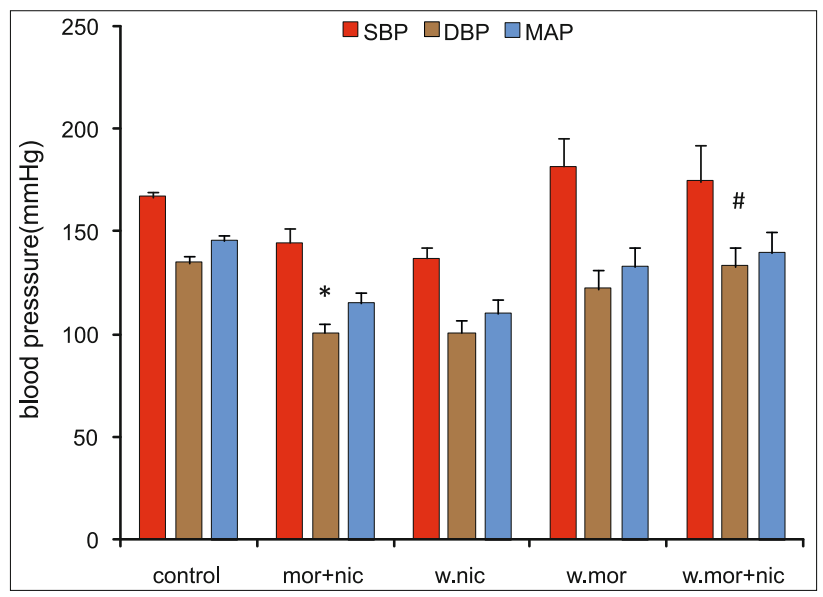

Fig. 1. Demonstrates changes in Systolic blood pressure, diastolic blood pressure and mean arterial pressure levels in pre-addict, postaddict and after withdrawal in hypertensive rats. SBP - systolic blood pressure, DBP - diastolic blood pressure, MAP - mean arterial pressure, mor+nic - morphine and nicotine treated rats, w. nic - nicotine treated rats that undergoes withdraw from nicotine, w. mor morphine treated rats that undergoes withdraw from morphine, $w$. mor+nic - morphine and nicotine treated rats that undergoes withdraw from nicotine and morphine. * significant difference in DBP between mor. nic with control group $(p<0.05)$, \# significant difference in DBP between w. mor+nic with w. nic and mor + nic groups $(\mathbf{p}<0.05)$. 


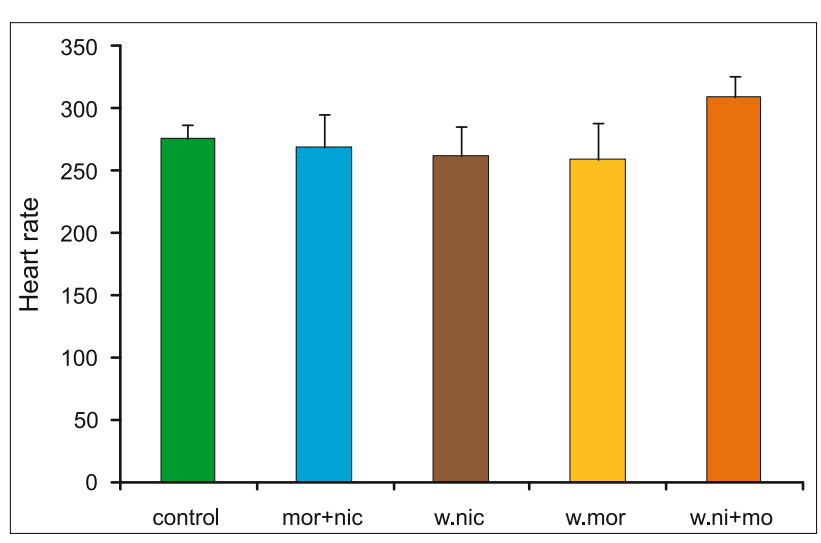

Fig. 2. Demonstrates changes in HR pre-addict, post-addict and after withdrawal in hypertensive rats. HR was not significantly differed with in groups. Other notations are the same as Fig. 1.

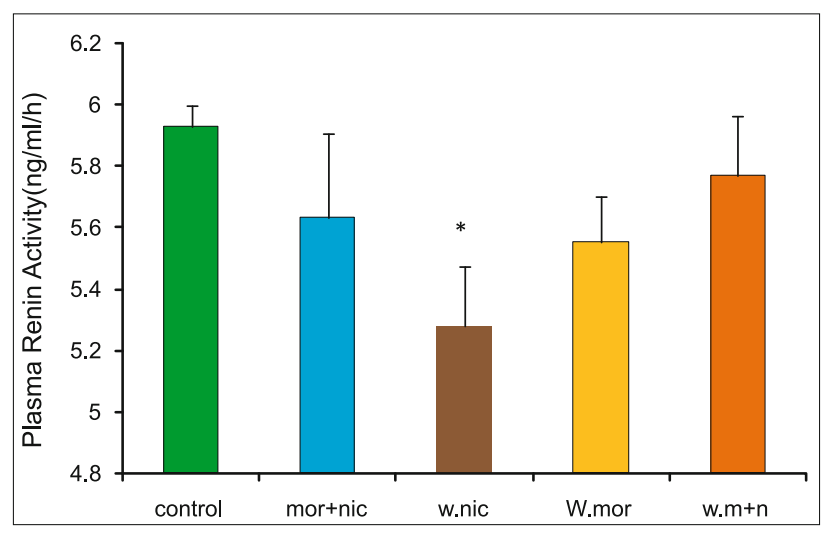

Fig. 3. Demonstrates changes in PRA pre-addict, post-addict and after withdrawal in hypertensive rats. * significant difference in PRA between win. nic with control group $(p<0.05)$. Other notations are the same as Fig. 1.

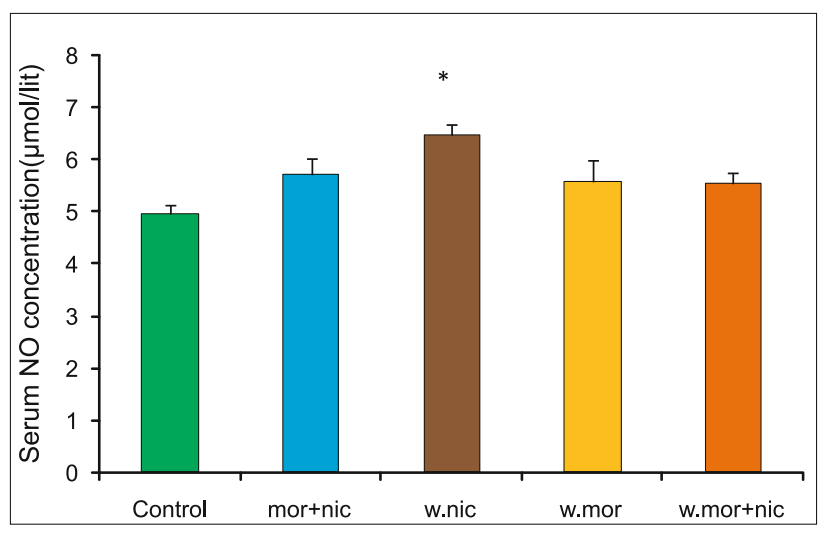

Fig. 4. Demonstrates the serum NO concentration, pre-addict, postaddict and after withdrawal in hypertensive rats. $*$ significant difference in serum NO concentration between win. nic with control group $(p<0.05)$. Other notations are the same as Fig. 1.

\section{Serum NO concentration}

The serum NO concentration in co-administration group (morphine and nicotine) was not different compared to control. But

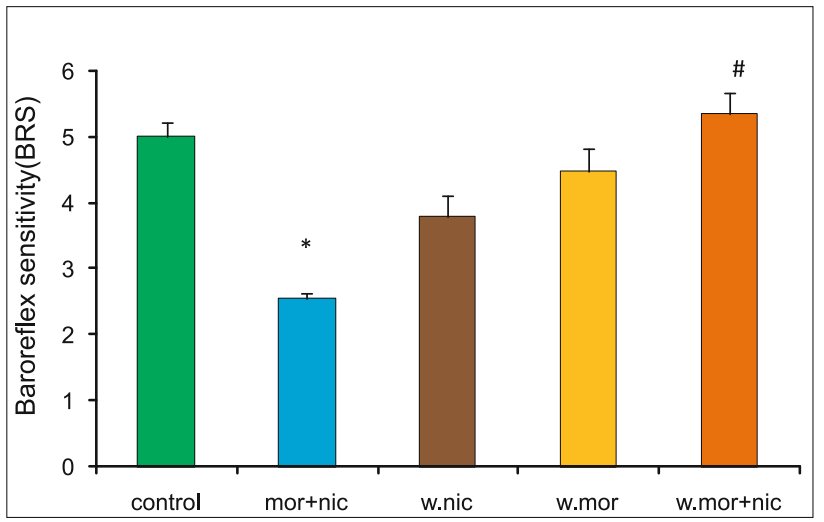

Fig. 5. Demonstrates the baroreflex sensitivity, pre-addict, post-addict and after withdrawal in hypertensive rats. * significant difference in BRS between w. nic with control and w. mor+nic; w. mor and $w$. nicgroups $(p<0.05)$, \# significant difference in BRS between mor+nic with control and $w$. nicgroups $(p<0.05)$. Other notations are the same as Fig. 1.

serum NO concentrationwas increased in nicotine withdrawal animals compared to control ( $\mathrm{p}<0.05)$ (Fig. 4).

\section{BRS}

In hypertensive rats that received contemporary of morphine and nicotine during 8 weeks, BRS was decrees compared to control, morphine withdrawal and morphine + nicotine withdrawal and nicotine withdrawal $(\mathrm{p}<0.05)$ and addict withdrawal specific in morphine + nicotine withdrawal compared to nicotine withdrawal completely reversed BRS to control group level $(\mathrm{p}<0.05)$ (Fig. 5).

\section{Discussion}

Previous investigations revealed that analysis of arterial pressure and HR are both important indices of baroreflex sensitivity $(24,25)$. In the present report we used cardiovascular markers including BP and HR up to provide a means of objective and continuous assessment effects of the co-administration of morphine and nicotine and those withdrawal on the BRS.

Current results evidently showed that blood pressure was significantly reduced in morphine and nicotine addicted hypertensive rats. The addiction withdrawal particular in morphine + nicotine withdrawal completely reversed blood pressure to control level, while during nicotine withdrawal the BP remained at low level. But it did not significantly affected HR.

Likewise the previous reports reduced BP following prolonged application of low-dose morphine in 2K1C animals might probably be due to decreased PRA levels (26). Some other research teams claimed that administration of chronic morphine decreases baseline cardiovascular parameters MAP and HR. following morphine withdrawal, and naloxone injection in morphine-treated rats, an increased MAP, HR (27), SBP and DBP was observed $(28,29)$. This was also occurred in case of nicotine consumption and increased $\mathrm{HR}, \mathrm{SBP}, \mathrm{DBP}$, and MAP is visible (30). Increase BP after administration of nicotine may be related to increase PRA level (31). 
The BRS is an independent risk factor for morbidity and major adverse cardiovascular event in patients suffering hypertension (32) and it appears to be an integral predictor for future cardiovascular events in patients with arterial hypertension (33). Evidences are in favors of the fact that hypertension is connected with decreased BRS $(32,34)$ and a significant negative correlation between presents BRS and BP (1).

We also evaluated BRS and found that morphine and nicotine addiction in hypertensive rats reduced BRS and morphine + nicotine withdrawal completely reversed it to the control level, while in case of nicotine withdrawal was remained at low level.

Serum NO levels were not differed between morphine and nicotine addicted hypertensive rats and it was sustained unchanged following control group, and addiction withdrawal.

Autonomous nervous dysfunction and inappropriately active sympathetic nervous system in patients with arterial hypertension is an important etiopathogenetic factor that contributes also to progression of disease, and the resultant cardiovascular risk. the BRS as a marker of autonomic dysfunction adding as a method for identifying patients at high cardiovascular risk (1).

Recent investigations described a relationship between NO and baroreflex function in hypertensive rats (35).

NOis an important biological modulator with diverse physiological activities that include vaso- relaxation (36-38). NO is derived from the action of NO synthase (NOS) on its substrate, $\mathrm{L}$-arginine. NO modulates vascular tone directly by relaxing vascular smooth muscle (39) and indirectly by restraining central sympathetic outflow (40) and NE release from nerve terminals (41). Overall, NO has been shown to have sym-central and peripheral mechanisms (42).

No have an important role in the autonomic nervous system regulating HR and especially, arterial pressure variability (43). NO had been postulated to be a modulator of sympathetic nerve activity, decreasing peripheral sympathetic nerve activity (44). It is proposed that NO can affect baroreflex via other factors. such as neural pathways (45) or pathophysiological conditions (44). These findings are in accordance with the paradigm that blood pressure variability is not the direct result of unbuffered variations in sympathetic discharge but, rather, is produced by an interaction between neural and humoral components (46).

Previous evidences in hypertensive rats offering a mechanism by which that neuronal NO production is impaired at a number of key central sites concerned with autonomic cardiovascular regulation (42).

Studies are also indicative that morphine affects peripheral or central BRS modulation (47). Endogenous and exogenous opioids regulate the performance of BRS throughout influencing central opioids receptors, including $\mathrm{Mu}$ and Kappa (48).

Other studies are demonstrating that acute and chronic injection of morphine significantly reduces SBP, DBP and MAP. Moreover, it significantly upregulates the sensitivity of baroreflexes (19). The BP increased more rapidly following administration of nicotine in hypertensive rats (47). So that nicotine causes induced BP and HR in parallel with impaired baroreflex sensitivity (15). In patients with cardiovascular disease who show- ing impaired BRS, such as patients with hypertension (49) and heart failure (50), impaired baroreflex function may leading significant tachycardia and sympathetic activation during cigarette smoking (51). Smoking of cigarette increases the activity of the autonomic nervous system (the sympathetic part), increases HR , BP and impairs the baroreflex $(52,53)$. The mechanism of increasing BP and HR by nicotine is believed to be regulated by activation of the sympathetic nervous system with a release of nor epinephrine and epinephrine (54),vasopressin release (55), or by affecting endothelial function (56). Chronic nicotine impairs endothelial function by decreasing the formation of $\mathrm{NO}$ and increasing the NO degradation via generation of free oxygen radicals (57) while nicotine cessation reverses smoking induced endothelial dysfunction (58). In concluding the BP and BRS are significantly reduced during morphine and nicotine addiction in hypertensive rat our proposed model however, serum NO level did failed to change. We found that withdrawal not improve serum NO concentration while BP and BRS achieved to its basal level pre administration of morphine and nicotine. Overall, with peripheral mechanisms could not modulate changes of BP and BRS. Thus, it seems that in addition to morphine and nicotine and those withdrawals other mechanisms could be involved in regulation of BP and BRS. Therefore, it might be suggested that during addiction and withdrawal number of key central sites concerned with autonomic cardiovascular regulation in regulation of $\mathrm{BP}$ and BRS is important.

\section{References}

1. Celovska D, Stasko J, Gonsorcik J, Diab A. The significance of baroreflex sensitivity in hypertensive subjects with stroke. Physiol Res 2009; 59 (4): 537-543.

2. Izzo JL, Jr, Taylor AA. The sympathetic nervous system and baroreflexes in hypertension and hypotension. Curr Hypertens Rep 1999; 1 (3): 254-263.

3. Liu AJ, Ma XJ, Shen FM, Liu JG, Chen H, Su DF. Arterial baroreflex: a novel target for preventing stroke in rat hypertension. Stroke 2007; 38 (6): 1916-1923.

4. Robinson TG, Dawson SL, Eames PJ, Panerai RB, Potter JF. Cardiac baroreceptor sensitivity predicts long-term outcome after acute ischemic stroke. Stroke 2003; 34 (3): 705-712.

5. Mancia G, Grassi G, Giannattasio C, Seravalle G. Sympathetic activation in the pathogenesis of hypertension and progression of organ damage. Hypertension 1999; 34 (4 Pt 2): 724-728.

6. Persson PB. Baroreflexes in hypertension: a mystery revisited. Hypertension 2005; 46 (5): 1095-1096.

7. Smart D, Lambert DG. The stimulatory effects of opioids and their possible role in the development of tolerance. Trends Pharmacol Sci 1996; 17 (7): 264-269.

8. Atweh SF, Kuhar MJ. Autoradiographic localization of opiate receptors in rat brain. II. The brain stem. Brain Res 1977; 129 (1): 1-12.

9. Uhl GR, Goodman RR, Kuhar MJ, Childers SR, Snyder SH. Immunohistochemical mapping of enkephalin containing cell bodies, fibers and nerve terminals in the brain stem of the rat. Brain Res 1979; 166 (1): 75-94. 
10. Grassi G, Seravalle G, Calhoun DA, Bolla GB, Giannattasio C, Marabini M et al. Mechanisms responsible for sympathetic activation by cigarette smoking in humans. Circulation 1994; 90 (1): 248-253.

11. Groppelli A, Giorgi DM, Omboni S, Parati G, Mancia G. Persistent blood pressure increase induced by heavy smoking. J Hypertens 1992; 10 (5): 495-499.

12. Trap-Jensen J, Carlsen JE, Svendsen TL, Christensen NJ. Cardiovascular and adrenergic effects of cigarette smoking during immediate non-selective and selective beta adrenoceptor blockade in humans. Eur J Clin Invest 1979; 9 (3): 181-183.

13. Eckberg DL, Cavanaugh MS, Mark AL, Abboud FM. A simplified neck suction device for activation of carotid baroreceptors. J Lab Clin Med 1975; 85 (1): 167-173.

14. Smyth HS, Sleight P, Pickering GW. Reflex regulation of arterial pressure during sleep in man. A quantitative method of assessing baroreflex sensitivity. Circ Res 1969; 24 (1): 109-121.

15. Mancia G, Groppelli A, Di Rienzo M, Castiglioni P, Parati G. Smoking impairs baroreflex sensitivity in humans. Am J Physiol 1997; 273 (3 Pt 2): H1555-1560.

16. LaCroix AZ, Lang J, Scherr P, Wallace RB, Cornoni-Huntley J, Berkman L et al. Smoking and mortality among older men and women in three communities. N Engl J Med 1991; 324 (23): 1619-1625.

17. Minami J, Ishimitsu T, Matsuoka H. Effects of smoking cessation on blood pressure and heart rate variability in habitual smokers. Hypertension 1999; 33 (1 Pt 2): 586-590.

18. Valenti VE, Ferreira C, Meneghini A, Ferreira M, Murad N, Ferreira Filho $\mathrm{C}$ et al. Evaluation of baroreflex function in young spontaneously hypertensive rats. Arq Bras Cardiol 2009; 92 (3): 205-215.

19. Shanazari AA, Aslani Z, Ramshini E, Alaei H. Acute and chronic effects of morphine on cardiovascular system and the baroreflexes sensitivity during severe increase in blood pressure in rats. ARYA Atheroscler Fall; 7 (3): 111-117.

20. El-Mas MM, El-Gowilly SM, Fouda MA, Saad EI. Role of adenosine A2A receptor signaling in the nicotine-evoked attenuation of reflex cardiac sympathetic control. Toxicol Appl Pharmacol 2001; 254 (3): 229-37.

21. Nematbakhsh M, Khazaei M. The effect of estrogen on serum nitric oxide concentrations in normotensive and DOCA Salt hypertensive ovariectomized rats. Clin Chim Acta 2004; 344 (1-2): 53-57.

22. Sato MA, Menani JV, Lopes OU, Colombari E. Commissural NTS lesions and cardiovascular responses in aortic baroreceptor-denervated rats. Hypertension 1999; 34 (4 Pt 2): 739-743.

23. Abdala AP, Haibara AS, Colombari E. Cardiovascular responses to substance $\mathrm{P}$ in the nucleus tractus solitarii: microinjection study in conscious rats. Am J Physiol Heart Circ Physiol 2003; 285 (2): H891-898.

24. Cerutti C, Barres C, Paultre C. Baroreflex modulation of blood pressure and heart rate variabilities in rats: assessment by spectral analysis. Am J Physiol 1994; 266 (5 Pt 2): H1993-2000.

25. deBoer RW, Karemaker JM, Strackee J. Hemodynamic fluctuations and baroreflex sensitivity in humans: a beat-to-beat model. Am J Physiol 1987; 253 (3 Pt 2): H680-689.

26. Delle M, Ricksten SE, Haggendal J, Olsson K, Skarphedinsson JO, Thoren P. Regional changes in sympathetic nerve activity and baroreceptor reflex function and arterial plasma levels of catecholamines, renin and vasopressin during naloxone-precipitated morphine withdrawal in rats. $\mathrm{J}$ Pharmacol Exp Ther 1990; 253 (2): 646-654.
27. Almela P, Atucha NM, Milanes MV, Laorden ML. Cross-talk between protein kinase A and mitogen-activated protein kinases signalling in the adaptive changes observed during morphine withdrawal in the heart. $J$ Pharmacol Exp Ther 2009; 330 (3): 771-782.

28. Buccafusco JJ. Cardiovascular changes during morphine withdrawal in the rat: effects of clonidine. Pharmacol Biochem Behav 1983; 18 (2): 209-215.

29. Chan R, Irvine R, White J. Cardiovascular changes during morphine administration and spontaneous withdrawal in the rat. Eur J Pharmacol 1999; 368 (1): 25-33.

30. Al-Kubati M, Al-Kubati AS, al'Absi M, Fiser B. The short-term effect of water-pipe smoking on the baroreflex control of heart rate in normotensives. Auton Neurosci 2006; 126-127: 146-149.

31. Balakumar P, Kaur J. Is nicotine a key player or spectator in the induction and progression of cardiovascular disorders? Pharmacol Res 2009; 60 (5): 361-368.

32. Ormezzano O, Cracowski JL, Quesada JL, Pierre H, Mallion JM, Baguet JP. Evaluation of the prognostic value of BARoreflex sensitivity in hypertensive patients: the EVABAR study. J Hypertens 2008; 26 (7): 1373-1378.

33. Lantelme P, Khettab F, Custaud MA, Rial MO, Joanny C, Gharib C et al. Spontaneous baroreflex sensitivity: toward an ideal index of cardiovascular risk in hypertension? J Hypertens 2002; 20 (5): 935-944.

34. Head GA. Cardiac baroreflexes and hypertension. Clin Exp Pharmacol Physiol 1994; 21 (10): 791-802.

35. Waki H, Murphy D, Yao ST, Kasparov S, Paton JF. Endothelial NO synthase activity in nucleus tractus solitarii contributes to hypertension in spontaneously hypertensive rats. Hypertension 2006; 48 (4): 644-650.

36. Marletta MA. Nitric oxide: biosynthesis and biological significance. Trends Biochem Sci 1989; 14 (12): 488-492.

37. Moncada S, Higgs EA. Molecular mechanisms and therapeutic strategies related to nitric oxide. Faseb J 1995; 9 (13): 1319-1330.

38. Kone BC. Nitric oxide in renal health and disease. Am J Kidney Dis 1997; 30 (3): 311-333.

39. Vallance P, Collier J, Moncada S. Effects of endothelium-derived nitric oxide on peripheral arteriolar tone in man. Lancet 1989; 2 (8670): 997-1000.

40. Hansen J, Jacobsen TN, Victor RG. Is nitric oxide involved in the tonic inhibition of central sympathetic outflow in humans? Hypertension 1994; 24 (4): 439-444.

41. Schuman EM, Madison DV. Nitric oxide and synaptic function. Annu Rev Neurosci 1994; 17: 153-183.

42. Chowdhary S, Townend JN. Nitric oxide and hypertension: not just an endothelium derived relaxing factor! J Hum Hypertens 2001; 15 (4): 219-227.

43. Lacchini S, Ferlin EL, Moraes RS, Ribeiro JP, Irigoyen MC. Contribution of nitric oxide to arterial pressure and heart rate variability in rats submitted to high-sodium intake. Hypertension 2001; 38 (3): 326-331.

44. Ma R, Zucker IH, Wang W. Reduced NO enhances the central gain of cardiac sympathetic afferent reflex in dogs with heart failure. Am J Physiol 1999; 276 (1 Pt 2): H19-26.

45. Castellano M, Rizzoni D, Beschi M, Muiesan ML, Porteri E, Bettoni G et al. Relationship between sympathetic nervous system activity, baroreflex and cardiovascular effects after acute nitric oxide synthesis inhibition in humans. J Hypertens 1995; 13 (10): 1153-1161. 


\section{$743-748$}

46. Akselrod S, Gordon D, Madwed JB, Snidman NC, Shannon DC, Cohen RJ. Hemodynamic regulation: investigation by spectral analysis. Am J Physiol 1985; 249 (4 Pt 2): H867-875.

47. Weksler-Zangen S, Chorev M, Weinstock M. Contrasting influences of central and peripheral opioids on cardiac baroreflex sensitivity in rabbits. J Cardiovasc Pharmacol 1992; 20 (5): 688-693.

48. Thurston CL, Starnes A, Randich A. Changes in nociception, arterial blood pressure and heart rate produced by intravenous morphine in the conscious rat. Brain Res 1993; 612 (1-2): 70-77.

49. Matsukawa T, Gotoh E, Hasegawa O, Shionoiri H, Tochikubo O, Ishii M. Reduced baroreflex changes in muscle sympathetic nerve activity during blood pressure elevation in essential hypertension. J Hypertens 1991; 9 (6): 537-542.

50. Ferguson DW, Berg WJ, Roach PJ, Oren RM, Mark AL. Effects of heart failure on baroreflex control of sympathetic neural activity. Am J Cardiol 1992; 69 (5): 523-531.

51. Narkiewicz K, van de Borne PJ, Hausberg M, Cooley RL, Winniford MD, Davison DE et al. Cigarette smoking increases sympathetic outflow in humans. Circulation 1998; 98 (6): 528-534.

52. Gerhardt U, Hans U, Hohage H. Influence of smoking on baroreceptor function: $24 \mathrm{~h}$ measurements. J Hypertens 1999; 17 (7): 941-946.
53. Lucini D, Bertocchi F, Malliani A, Pagani M. Autonomic effects of nicotine patch administration in habitual cigarette smokers: a doubleblind, placebo-controlled study using spectral analysis of RR interval and systolic arterial pressure variabilities. J Cardiovasc Pharmacol 1998; 31 (5): 714-720.

54. Cryer PE, Haymond MW, Santiago JV, Shah SD. Norepinephrine and epinephrine release and adrenergic mediation of smoking-associated hemodynamic and metabolic events. N Engl J Med 1976; 295 (11): 573-577.

55. Waeber B, Schaller MD, Nussberger J, Bussien JP, Hofbauer KG, Brunner HR. Skin blood flow reduction induced by cigarette smoking: role of vasopressin. Am J Physiol 1984; 247 (6 Pt 2): H895-901.

56. Celermajer DS, Sorensen KE, Georgakopoulos D, Bull C, Thomas $\mathbf{O}$, Robinson $\mathbf{J}$ et al. Cigarette smoking is associated with dose-related and potentially reversible impairment of endothelium-dependent dilation in healthy young adults. Circulation 1993; 88 (5 Pt 1): 2149-2155.

57. Toda N, Toda H. Nitric oxide-mediated blood flow regulation as affected by smoking and nicotine. Eur J Pharmacol 15; 649 (1-3): 1-13.

58. Powell JT. Vascular damage from smoking: disease mechanisms at the arterial wall. Vasc Med 1998; 3 (1): 21-28.

Received May 24, 2013. Accepted November 26, 2013. 